\title{
Populational equilibrium through exosome-mediated Wnt signaling in tumor progression of diffuse large B-cell lymphoma
}

\author{
Raphael Koch, ${ }^{1}$ Martin Demant, ${ }^{1}$ Thiha Aung, ${ }^{1}$ Nina Diering, ${ }^{1}$ Anna Cicholas, ${ }^{1}$ Bjoern Chapuy, ${ }^{2}$ Dirk Wenzel, ${ }^{3}$ \\ Marlen Lahmann, ${ }^{1}$ Annemarie Güntsch, ${ }^{1}$ Christina Kiecke, ${ }^{1}$ Sabrina Becker, ${ }^{1}$ Timo Hupfeld, ${ }^{1}$ Vivek Venkataramani, ${ }^{1}$ \\ Marita Ziepert, ${ }^{4}$ Lennart Opitz, ${ }^{5}$ Wolfram Klapper, ${ }^{6}$ Lorenz Trümper, ${ }^{1}$ and Gerald G. Wulf ${ }^{1}$ \\ ${ }^{1}$ Department of Hematology and Oncology, Georg-August-University, Goettingen, Germany; ${ }^{2}$ Dana-Farber Cancer Institute, Boston, MA; ${ }^{3}$ Max-Planck \\ Institute for Biophysical Chemistry, Goettingen, Germany; ${ }^{4}$ Institute for Medical Informatics, Statistics and Epidemiology, Leipzig, Germany; ${ }^{5}$ Functional \\ Genomics Center Zurich, Zurich, Switzerland; and ${ }^{6}$ Pathology, Hematopathology Section and Lymph Node Registry, Kiel, Germany
}

\section{Key Points}

- Diffuse large B-cell lymphomas are composed of clonogenic side population (SP) cells and non-SP cells organized in a dynamic equilibrium.

- Exosome-mediated Wnt signaling modulates transitions of cell states and tumor progression amenable to drug targeting.
Tumors are composed of phenotypically heterogeneous cell populations. The nongenomic mechanisms underlying transitions and interactions between cell populations are largely unknown. Here, we show that diffuse large B-cell lymphomas possess a self-organized infrastructure comprising side population (SP) and non-SP cells, where transitions between clonogenic states are modulated by exosomemediated Wnt signaling. DNA methylation modulated SP-non-SP transitions and was correlated with the reciprocal expressions of Wnt signaling pathway agonist Wnt3a in SP cells and the antagonist secreted frizzled-related protein 4 in non-SP cells. Lymphoma SP cells exhibited autonomous clonogenicity and exported Wnt3a via exosomes to neighboring cells, thus modulating population equilibrium in the tumor. (Blood. 2014;123(14):2189-2198)

\section{Introduction}

Although tumor cells arise from the malignant transformation of a single cell, their samples are inherently composed of heterogeneous tumor cell populations, as evidenced by differential marker expression or differences in cellular behavior. ${ }^{1}$ Beyond acquisition of genetic mutations during tumor progression, this heterogeneity may also be due to nongenomic alterations, such as the induction of epigenetic alterations associated with extrinsic cues. ${ }^{2}$ One such phenotypic variation includes the confinement of clonogenic growth to only few tumor cells, ${ }^{3}$ eventually leading to the concept of cancer stem cells (CSCs). ${ }^{4-6}$ One approach to isolate CSCs has been proposed by adapting the Hoechst 33342 staining method to purify hematopoietic stem cells (HSCs) as side population (SP) cells. $^{7-11}$

Wnt signal-transduction pathways represent an essential module in the morphogenesis of all metazoa. ${ }^{12}$ Originally described in Drosophila, Wnt proteins mediate communication between cells over a short distance, where concentration gradients play an important role as signals directing differentiation and tissue homeostasis. ${ }^{13}$ Perturbations of Wnt signaling have been described during malignant transformation, particularly in colorectal cancer and melanoma. ${ }^{14,15}$ In acute leukemia, a canonical Wnt signaling agonist, Wnt3a, was found to act as a hematopoietic growth factor, supporting disease progression even in the absence of mutations in Wnt pathway components. ${ }^{16-18}$

The hydrophobic nature of Wnt proteins acquired as a result of extensive posttranslational processing ${ }^{19,20}$ necessitates specific carrier mechanisms for their export from the endoplasmic reticulum to the cell surface. The extracellular transport of Wnt proteins through both specific lipoprotein carrier proteins and their association with the lipid membranes of vesicular structures has been described in Drosophila. ${ }^{21-25}$ Such secretory vesicles of Drosophila resemble exosomes, which have also been identified in multiple human cell systems. Exosomes are microvesicular structures with a mean size of 50 to $100 \mathrm{~nm}$, released by exocytosis following intracellular assembly in multivesicular bodies (MVBs) ${ }^{26}$ In normal physiology, exosomes are secreted from erythroid progenitors during progenitor cell maturation, as well as from normal and transformed B lymphocytes and dendritic cells, and possess immunomodulatory functions. ${ }^{27-29}$

In view of the above and the emerging role of exosomes in the Wnt signaling pathway, the objective of the present study was to identify putative tumor stem cells in aggressive lymphomas of
Submitted August 27, 2013; accepted January 11, 2014. Prepublished online as Blood First Edition paper, February 21, 2014; DOI 10.1182/blood-2013-08523886.
The publication costs of this article were defrayed in part by page charge payment. Therefore, and solely to indicate this fact, this article is hereby marked "advertisement" in accordance with 18 USC section 1734.

R.K. and M.D. contributed equally to this study. 
diffuse large B-cell lymphoma (DLBCL) type and investigate the mechanisms by which the formation of SP and non-SP cells are modulated.

\section{Materials and methods}

\section{Cells, antibodies, small molecules, plasmids, and vectors}

The previously described DLBCL cell lines SUDHL4, U2932, and Karpas 422 were obtained from a public depository (DSMZ, Braunschweig, Germany), the cell line Balm- $3^{30}$ was kindly provided by B. Glass, and the cell lines OCI Ly1 and OCI Ly $3^{31,32}$ were obtained from the Ontario Cancer Institute. The cell lines L-Wnt3a (ATCC \#CRL-2647) and L cells (ATCC \#CRL-2648) were kindly provided by T. Pukrop. Approval for the collection and analysis of patient samples after informed consent was obtained from the institutional review board of the University Medicine Goettingen and was conducted according to the Declaration of Helsinki. For propagation of human lymphoma cells in ovo, fertilized chick eggs were incubated at $37.8^{\circ} \mathrm{C}$ and $80 \%$ relative humidity and lymphoma cells applied on the chorioallantoic membrane (CAM) on day 10 (for details, see supplemental "Experimental procedures"). For SP cell analysis or sorting, the mononuclear cells were stained with the fluorescent dye Hoechst 33342 (Sigma-Aldrich) at a concentration of $5 \mathrm{mg} / \mathrm{mL}$ ( $37^{\circ} \mathrm{C}$ for 2 hours) as previously described. ${ }^{7}$ Exosomes were prepared by differential centrifugation according to standard protocols (for details, see supplemental "Experimental procedures"). ${ }^{28}$ Small molecules were used at the concentrations indicated (XAV939, SB216763, IWR1, and IWP2; all from Sigma-Aldrich). For silencing Wnt3a and secreted frizzled-related protein 4 (SFRP4), validated specific small hairpin RNA sequences were used (The RNAi Consortium, www.broadinstitute.org/rnai/trc; shWnt3a: shWnt3a I, TRCN0000089120; shWnt3a II, TRCN0000089122; shSFRP4, clone TRCN0000014490) and were cloned into pLKO.1-eGF (Addgene), and lentiviral particles produced in a human embryonic kidney $293 \mathrm{~T}$ cell producer cell line with the plasmids pCMV- $\Delta$ R8.91 (containing gag, pol, and rev genes) and pMD.G (VSV-G-expressing plasmid) following standard protocols. Green fluorescent protein (GFP)-positive variants of the cell lines OCI Ly3 and OCI Ly1 were generated by lentiviral transduction. GFP expression over time was routinely screened in parallel to all experiments by fluorescence-activated cell sorter, documenting stable GFP expression $>98 \%$ in the transduced variants (supplemental Figure 3c).

\section{Gene expression, DNA methylation, microscopy, sodium dodecyl sulfate polyacrylamide gel electrophoresis, western blot, and PCR}

For gene expression analysis, $300 \mathrm{ng}$ of purified RNA was labeled with biotin according to the Affymetrix Gene Human Arrays 1.0 ST protocol (details provided in supplemental "Experimental procedures"). The HumanMethylation27 BeadChip (Illumina, San Diego, CA) uses Infinium technology, previously described for SNP genotyping, ${ }^{33}$ to perform genome-wide screening of DNA methylation patterns (details provided in supplemental "Experimental procedures"). Confocal microscopy, immunoelectron microscopy according to the Tokuyasu method, sodium dodecyl sulfate polyacrylamide gel electrophoresis, western blot, and polymerase chain reaction (PCR) were performed according to standard protocols (details provided in supplemental "Experimental procedures").

\section{Statistical evaluations}

The indicated statistical tests were performed using GraphPad Prism, version 4.03 for Windows (GraphPad Software, San Diego, CA; www.graphpad. com), and $P$ values are given for each experiment. Error bars represent standard deviations of samples.

\section{Results}

Role of SP cells in context-dependent regulation of clonogenicity and population homeostasis in DLBCL

The identification of stem cells with SP phenotype in several cancers prompted us to explore for such cells in DLBCL. We found SP cells as a regular constituent of DLBCL cell lines, at mean proportions ranging from $0.09 \%$ to $2.9 \%$ (Figure 1A-B). The proportions of SP cells were characteristic for individual cell lines, and these cells did not segregate with cell lines of either activated B-cell (ABC)-type origin (U2932 and OCI Ly3) or germinal center B-cell (GCB)-type origin (SUDHL4, Karpas 422, Balm-3, and OCI Ly1). Although SP cell number variability was observed between repetitive measurements of the same cell line, the formation of SP cells was also found after in vivo propagation either in ovo or in the mouse (Figure 1A). Importantly, we also identified SP cells in primary tumors of 8 untreated patients with DLBCL at a mean proportion of $0.6 \%$ (range, $0.15 \%$ to $2.06 \% ; \mathrm{n}=8$ ), wherein the SP cells harbored the clonal markers of mature B cells of the main lymphoma cell populations (Figure 1B-E). Further phenotypic analysis revealed no major differences in cell surface marker expression and, in particular, no differences in HSC marker expression between SP and nonSP cells (supplemental Figures 4c and 5). Lymphoma SP cells expelled doxorubicin more efficiently than non-SP cells, whereas the expression levels of ABC transporter G2 in SP and non-SP cells were similar (Figure $1 \mathrm{G}$ and supplemental Figure 4a). ${ }^{34}$ Noteworthy, SP cells of all cell lines were enriched for cells in the $G_{0} / G_{1}$ cell-cycle phase (Figure 1G). Previous results have shown that malignant SP cells gave rise to progeny of both SP and non-SP cell phenotypes, whereas non-SP cell fractions were mostly incompetent to continue cancer cell propagation. ${ }^{9}$ This finding was confirmed in DLBCL cell lines (supplemental Figure 1), and a colony-forming unit (CFU) assay was performed to analyze clonogenicity of purified SP and non-SP cells. Colony formation was restricted to SP cells in all cell lines, with many colonies arising from a few SP cells compared with a complete absence or the presence of a few colonies from high numbers of non-SP cells (Figure 2A-B and supplemental Figure 2). Similarly, implantation of purified SP and non-SP cells onto the CAM of embryonated chicken eggs resulted in the formation of gross tumors from all the 11 implants of $5 \times 10^{6} \mathrm{SP}$ cells, whereas equal numbers of non-SP cells produced no or significantly smaller tumors (Figure 2C-D). To more closely mimic the in vivo situation of densely packed lymphoma cells in the tumor tissue, we generated GFP-positive variant cell lines by lentiviral transduction and mixed populations of GFP-labeled SP and unlabeled non-SP cells from the OCI Ly3 cell line in a ratio of 4 SP cells to 96 nonSP cells (supplemental Figure 3c). We observed a relative expansion of progeny from labeled SP cells in the growing tumor, accompanied by an expansion of progeny from unlabeled non-SP cells (Figure 2E). This observation in OCI Ly3 was confirmed in cell line OCI Ly1 (Figure 2F). This finding refutes our initial expectation that there would be a growth of only SP cell progeny. In addition, both SP and non-SP fractions of the growing tumor were composed of equal amounts of labeled and unlabeled cells, indicating that SP cells had generated non-SP cells as expected; however, non-SP cells also had generated both SP and non-SP cells (Figure 2E-F). In the tumor tissue, the progeny of the labeled SP cells were evenly distributed among the unlabeled cells, suggesting that the generation of progeny from SP and non-SP cells did not follow a clonal pattern, but rather occurred as diffusely admixed cells (Figure 2G). Therefore, lymphoma 

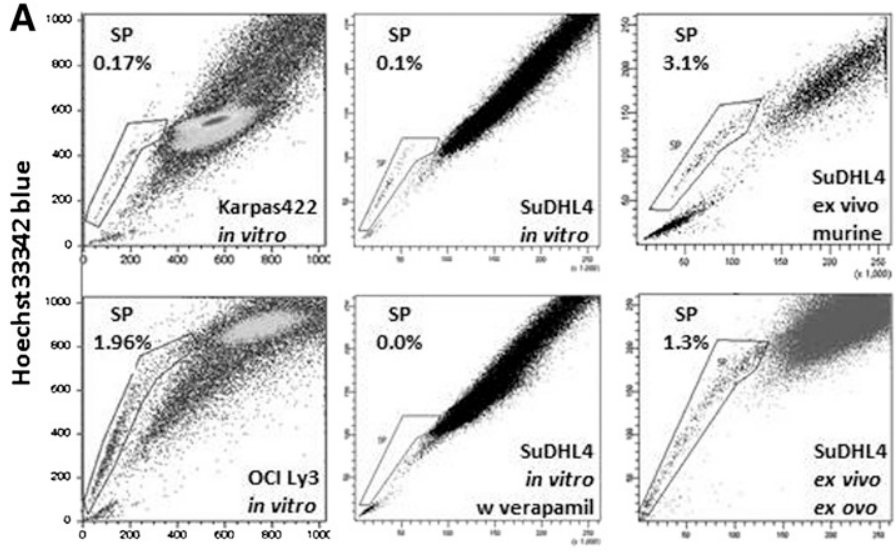

Hoechst 33342 rec
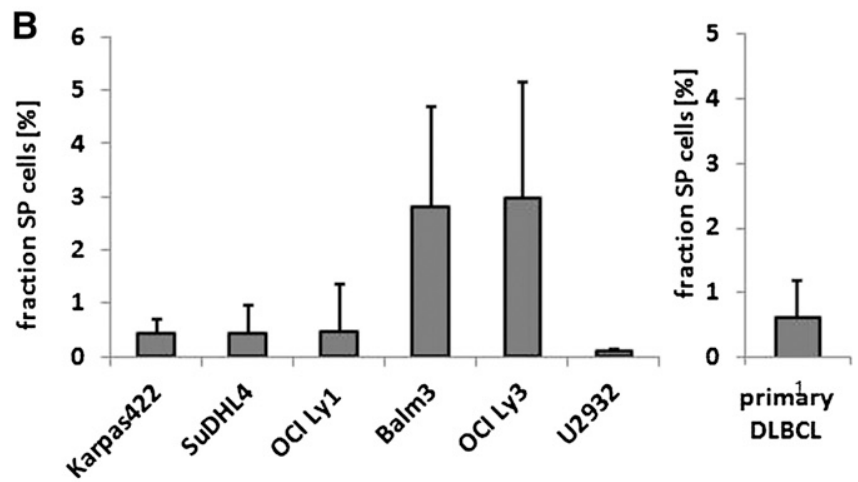
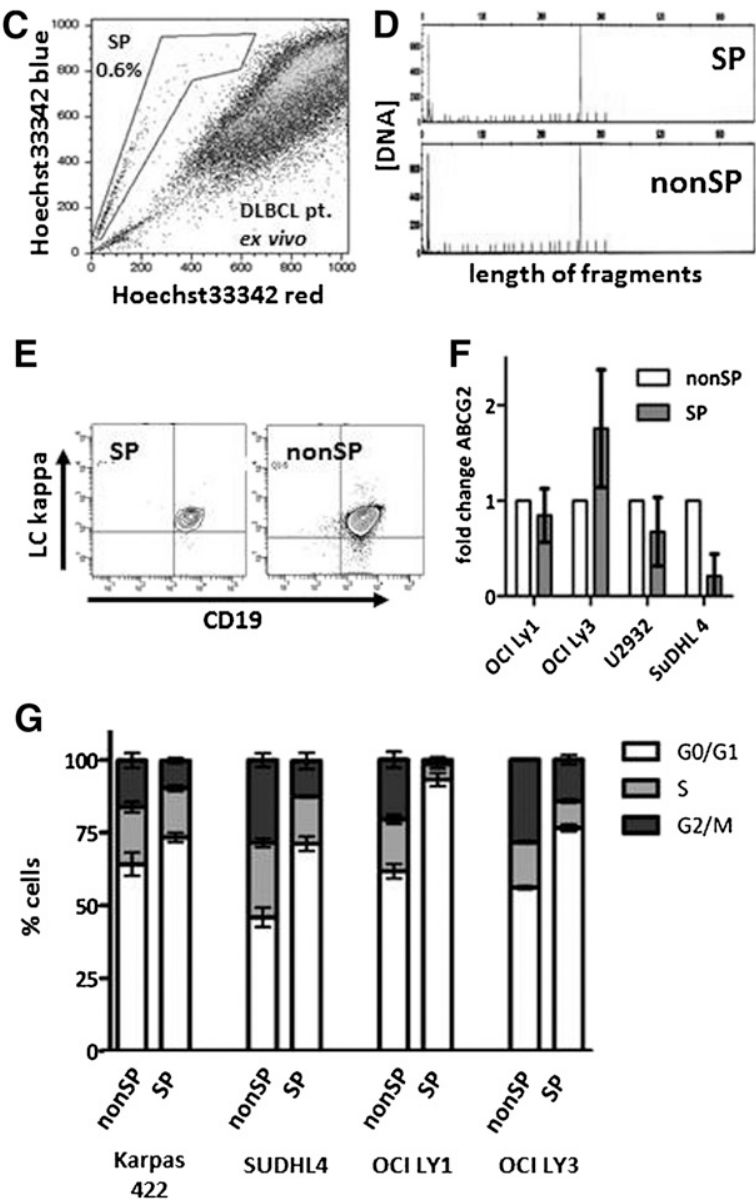

Figure 1. SP cells in DLBCL. (A) Hoechst 33342 staining detected an SP (as boxed) with low dye content in lymphoma cell lines both in vitro and in vivo after passaging by xenotransplantation in the mouse or egg, as indicated. Coincubation with verapamil $(50 \mu \mathrm{g} / \mathrm{mL})$ abrogated SP cell formation (lower middle panel); the proportions of SP cells are given in percentage of all nucleated cells. (B) The proportions of SP cells were characteristic for each cell line of ABC type (OCI Ly3 and U2932) and GCB type (Karpas 422, SuDHL4, OCI Ly1, and Balm-3; $n=10$ per cell line; standard deviations indicated by errors bars). (C) SP cells were also detected in primary patient lymphoma cell specimen (example in C; mean value from 8 patient samples in $\mathrm{B}$, separate column). (D) Identical monoclonal immunoglobulin heavy chain rearrangements were found in SP and non-SP cells, as shown for the representative patient sample of $C$. (E) Expression of CD19 and a low level of immunoglobulin $\kappa$ light chain in SP and non-SP cells, compatible with an identical mature B-cell phenotype in both cell types. (F) Comparable transcript levels for the ABC transporter G2 in the cell lines OCI Ly1, OCI Ly3, U2932, and SuDHL4. (G) Cell-cycle analysis of non-SP vs SP cells of the cell lines Karpas 422 , SuDHL4, OCI Ly1, and OCI Ly3 with significant enrichment of SP cells in the $\mathrm{G}_{0} / \mathrm{G}_{1}$ phase (2-way ANOVA $\mathrm{G}_{0} / \mathrm{G}_{1}$ non-SP vs SP all cell lines $P<.0001$, each cell line $\mathrm{n}=3$ ).

SP cells represent a regular constituent of aggressive B-cell lymphoma and have the capacity for clonogenic growth as single cells. As separated cells, clonogenic growth is restricted to SP cells. In admixed populations of a complete tumor, however, transitions from SP to non-SP cells as well as from non-SP to SP cells occur (Figure $2 \mathrm{H}$ ).

\section{Modulation of canonical Wnt signaling in DLBCL SP cells}

The observation that SP and non-SP cells act as transient functional lymphoma cell phenotypes raised our interest in the regulatory process involved. Global DNA hypomethylation has been recently recognized as a feature of HSCs, particularly those of the SP cell phenotype. ${ }^{35}$ Therefore, to determine whether DNA hypomethylation is involved in SP cell generation, the lymphoma cell lines were incubated with the DNA demethylating agent 5-aza-2'-deoxycytidine. It was observed that incubation augmented the fraction of SP cells in lymphoma cultures, particularly those of OCI Ly3, OCI Ly1, and Karpas 422 (supplemental Figure 6a-b). In order to explore candidate genes, we first applied a global gene promoter methylation analysis, which identified, among others, both Wnt and Wnt receptor signaling as differentially methylated functional genes classes (supplemental Table 1). To further narrow down those genes differentially expressed between SP and non-SP cells, we then performed a combined analysis, where genes with promoter hyper- or hypomethylation status were correlated with either a low or high transcript level, respectively. Promoter hypomethylation of SFRP4 was found to be associated with a low SFRP4 expression, whereas $W n t 3 a$ promoter hypomethylation was found to be correlated with an increased Wnt $3 a$ gene expression in OCI Ly3 SP cells (supplemental Figure 6c-d), and increased expression of Wnt $3 a$ as well as low levels of SFRP4 in SP cells was consistently found in the 4 DLBCL cell lines tested (Figure 3A). Apart from the OCI Ly3 cell line, Wnt3a and SFRP4 proteins were detected at variable expression levels in other lymphoma cell lines tested (Figure 3B), and western blot analyses of sorted cells confirmed high expression of Wnt $3 a$ vs low expression of SFRP4 in OCI Ly3 and OCI Ly1 SP cells (Figure 3C and supplemental Figure 3a-b). Next, we searched for $\beta$-catenin, because it represents a major signaling intermediate of canonical Wnt signaling activation. Expectedly, western blot analyses of whole-cell lysates confirmed the presence of $\beta$-catenin in all the lymphoma cell lines, with the highest levels of its expression in OCI Ly3 (Figure 3B). We found that a fraction of $\beta$-catenin was 

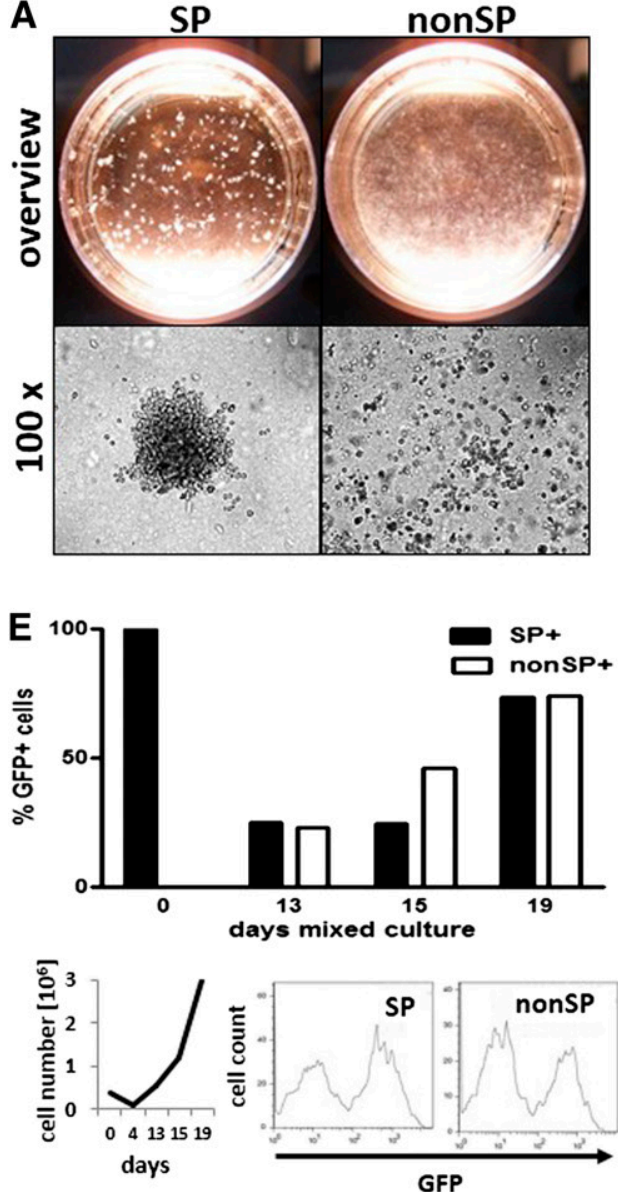

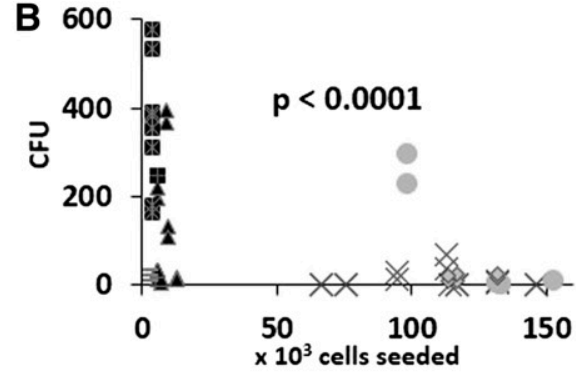

C

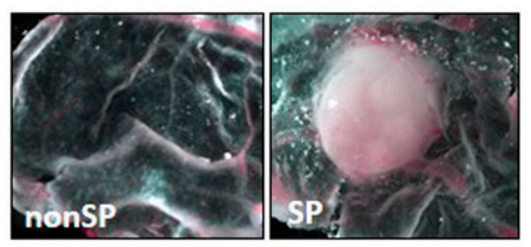

$\mathbf{F}$

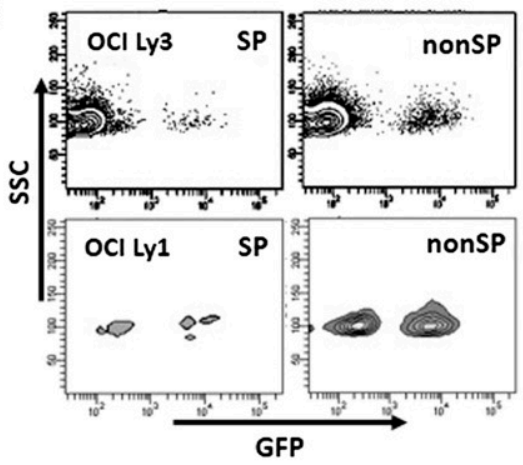

D

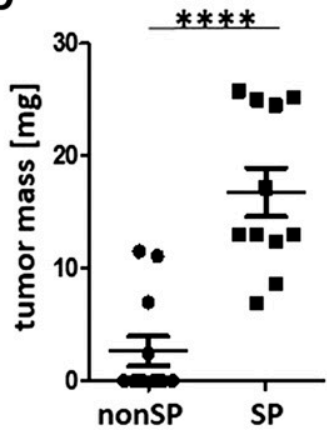

$\mathbf{G}$
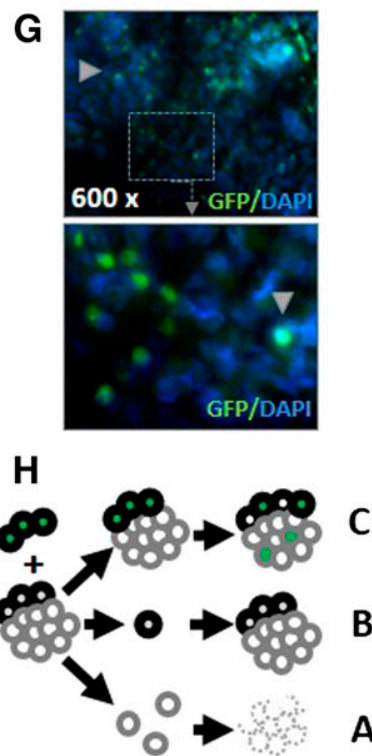

Figure 2. Lymphoma SP cells are enriched in clonogenic capacity and represent a transient phenotype. (A) Colony formation from purified lymphoma SP cells (CFUs), compared with no or only few colonies from corresponding non-SP cell preparations (representative example for Balm-3 survey, upper row; microscopic view, lower row). (B) For 5 cell lines, CFU activity was significantly enriched in the SP compared with non-SP cells (unpaired 2-sided $t$ test; data for each individual cell line; see supplemental Figure 2). (C-D) In vivo, $0.5 \times 10^{6}$ purified SP cells generated tumors within 8 days in a modified CAM assay in ovo, compared with no or significantly smaller tumors from equal amounts of non-SP cells (C, representative examples of tumors; $\mathrm{D}$, tumor mass; unpaired 2-sided Student $t$ test, ${ }^{* \star * \star} P<.001 ; \mathrm{n}=19$; cell line OCI Ly3). (E) After admixture of $4 \%$ GFP-positive SP cells to $96 \%$ unlabeled non-SP cells, contribution of GFP-positive cells to the whole tumor were measured by flow cytometry and microscopy in vitro and in vivo. In the course of tumor expansion (left lower row; expansion of all cells $\times 10^{6}$ ), the proportion of GFP-positive cells and of non-SP cells expanded, with the emergence of GFP-negative SP cells (upper row, example of flow cytometric GFP analysis of SP and non-SP at day 19; right lower row, representative results of 3 independent experiments). (F) After implantation of mixed cultures into the egg, GFP-positive SP cells contributed to both SP and non-SP cell fractions at day 8 of tumor growth in vivo, again with progeny from GFP-negative non-SP cells to both populations at day 8 in the cell line OCI Ly3 and OCI Ly1 (F, $n=2$ each). (G) In the OCI Ly3 tumors after in ovo passaging, GFP-positive cells (arrowheads) were distributed evenly between progeny of non-SP cells throughout the tumor tissue (immunofluorescence staining with an anti-GFP antibody, $n=10$; enlargement of insert $\times 8)$. $(H)$ Collectively, whereas isolated non-SP cells died off in culture or in the host (A) and isolated SP cells formed colonies (B), in mixed cultures both SP and non-SP cells contributed to tumor propagation (C).

localized in the cytoplasm, particularly the perinuclear region (supplemental Figure 7b), and that exogenic recombinant Wnt3a increased $\beta$-catenin levels in OCI Ly3 and OCI Ly1 cells (Figure 3D). A significantly higher proportion of $\beta$-catenin was detected in the nuclei of OCI Ly3 SP cells when compared with the proportion in the nuclei of non-SP cells (Figure 3E-F).

Subsequently, we suppressed Wnt3a expression by transfecting the OCI Ly3 lymphoma cells with lentiviral shWnt3a constructs (supplemental Figure 8). Silencing of Wnt3a expression significantly reduced the proliferation of OCI Ly3 cells in lymphoma cell suspension cultures (Figure 4A), and proliferation was completely inhibited in subsequent passages $(n=4)$. In the CFU assay, the shWnt3-transfected cell lines showed low clonogenic capacity, resulting in a few colonies of densely packed cells, when compared with that exhibited by the scattered colonies of mock-transduced OCI Ly3-type cells (Figure 4B-C). Further, the Wnt3a-knockdown variants produced, if any, only minimal tumors following implantation on the CAM in vivo (Figure 4E).
These findings were confirmed in vitro, wherein the purified SP cells of Wnt3a-knockdown variants exhibited low colony formation ability (Figure 4D). Subsequently, the nontransfected non-SP cells were combined with the SP cells of shWnt3a variants in a ratio of 96:4, and no significant CFU activity was observed (Figure 4D, third column), indicating that the loss of Wnt3a in the SP cells could not be compensated by the residual Wnt agonist expression in the non-SP cells. Taken together, these results provide evidence that Wnt3a originating from lymphoma SP cells is an essential factor for clonogenicity and proliferation of SP cells in the whole tumor population.

Vice versa, we also analyzed the influence of the differential expression of SFRP4 in SP and non-SP cells. All lymphoma cell lines expressed and secreted SFRP4 at variable levels (Figure 3B). Functionally, the addition of exogenous recombinant SFRP4 reduced clonogenic activity in all cell lines, which was in turn associated with diminished proportions of SP cells in the cell cultures, in contrast to the increased CFU activity of OCI Ly3 cells observed 


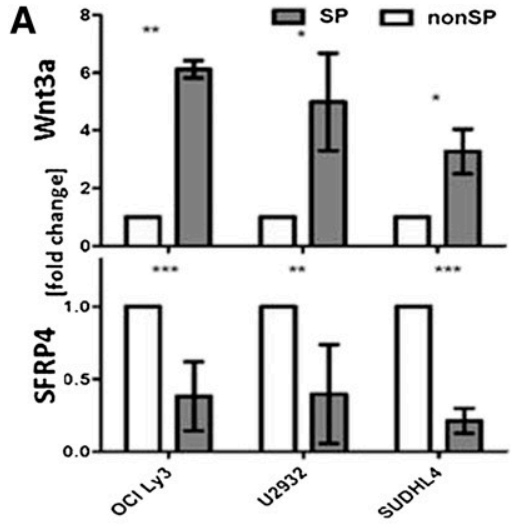

B
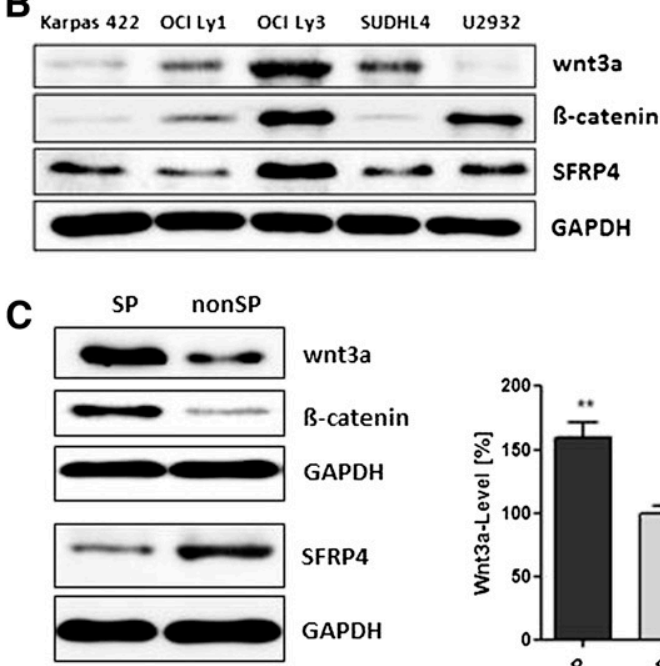
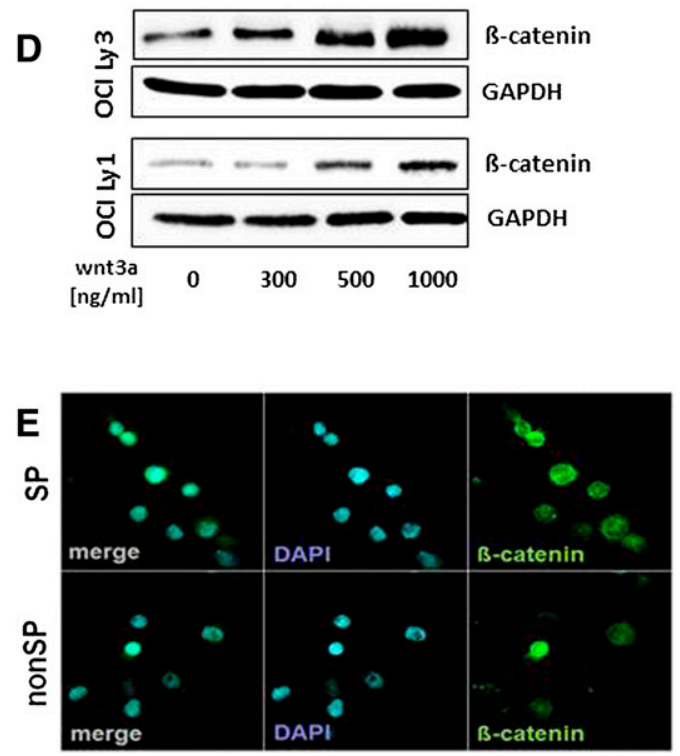

$\mathbf{F}$

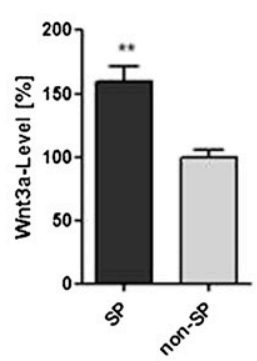

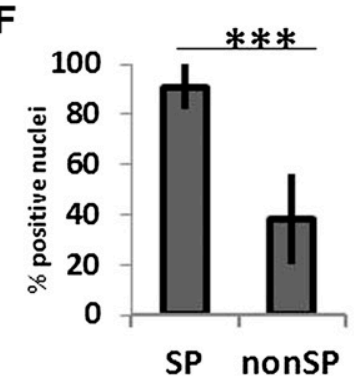

Figure 3. Activation of the canonical Wnt signaling in SP cells from DLBCL cell lines. (A) Increased expression of Wnt3a as well as reduced expression of SFRP4 was found in the SP vs non-SP cells of OCI Ly3 cells by quantitative reverse-transcription PCR (normalized to GAPDH; $n=10$ OCI Ly3, $n=5$ U2932, $n=3$ SUDLH4; unpaired Student $t$ test). (B) Western blot showed expression of Wnt3a and SFRP4 in all lymphoma cell lines tested, with variable expression levels. (C) Increased levels of Wnt3a and $\beta$-catenin vs lower levels of SFRP4 in whole-cell lysates of SP cells compared with non-SP cells of OCI Ly3. Densitometric quantification (right) of Wnt3a levels in SP and nonSP cells of OCI Ly3 ( $\mathrm{n}=4$, unpaired Student $t$ test, $P=.0047)$. (D) Cellular $\beta$-catenin in OCI Ly3 and OCI Ly1 cells was enhanced by exogenic stimulation with recombinant Wnt3a (concentration as indicated for 8 hours). (E) $\beta$-catenin localized to the nuclei of both SP and non-SP cells from OCI Ly3, with higher frequency in SP cells (F; $n=200$, OCl Ly3, $P<.0001$; paired 2-sided Student $t$ test). (G) C-myc and axin2 were expressed at higher levels in SP compared with non-SP cells in OCI Ly3 (axin2: $\mathrm{n}=5, P<.001$; c-myc: $\mathrm{n}=4, P<.01$; 2-way ANOVA).

upon the addition of a neutralizing antibody against SFRP4 (Figure 4F-G and supplemental Figure 8).

We applied several pharmacologic modulators of Wnt3amediated canonical Wnt signaling to OCI Ly3 cells in the CFU assay (Figure 5A). Although the agents had no effect on the rare CFUs from non-SP cells, we found significant effects on the clonogenic population of SP lymphoma cells. Whereas the recombinant Wnt3a and the GSK- $\beta$ inhibitor SB-216768 stimulated CFU formation from lymphoma SP cells, the neutralizing antibody against Wnt3a and the tankyrase inhibitor XAV939 produced inhibitory effects (Figure 5A). We then applied XAV939 to lymphoma xenografts in the CAM assay and discovered a dose-dependent reduction of tumor size by this agent (Figure 5B). Histologic dissection of XAV939-treated lymphomas further showed a significant loss of tumor cell density in the implants treated with an XAV939 concentration of $>1 \mathrm{mM}$ (Figure 5B and supplemental Figure 9). The effects of XAV939 were associated with a dose-dependent reduction of cellular $\beta$-catenin (Figure 5D). Notably, small molecules other than XAV939 acting as Wnt-signaling inhibitors also induced a reduction of the CFU activity in lymphoma cells, where IWP2 inhibited palmitoylation of Wnt and IWR1 increased axin2 protein levels (Figure 5C). Importantly, a significant reduction in the tumor CFU activity and cell growth by XAV939 was also observed in further lymphoma cell lines (supplemental Figure 9a). The effect of XAV939 was associated with decreased proportions of SP cells, which could be attributed to growth arrest rather than the direct cytotoxicity of XAV939 (Figure 5E and supplemental Figure S9b-c). Thus, Wnt3a appears to be an essential agonist of cell growth in DLBCL cell lines, which could be blocked by small molecules.

\section{Exosome-mediated transport of the Wnt3a protein}

For the extracellular transmission of lipophilic Wnt3a, several mechanisms have been discovered, including specific extracellular carrier lipoproteins as well as membranous vehicles termed argosomes. ${ }^{21-24}$ Our recent study demonstrated that aggressive lymphoma cells produce ample amounts of exosomes, which are involved in mechanisms of cellular resistance against humoral immunotherapy. ${ }^{29}$ In the present study, we applied high amounts of purified exosomes to lymphoma cells in the CFU assay and 

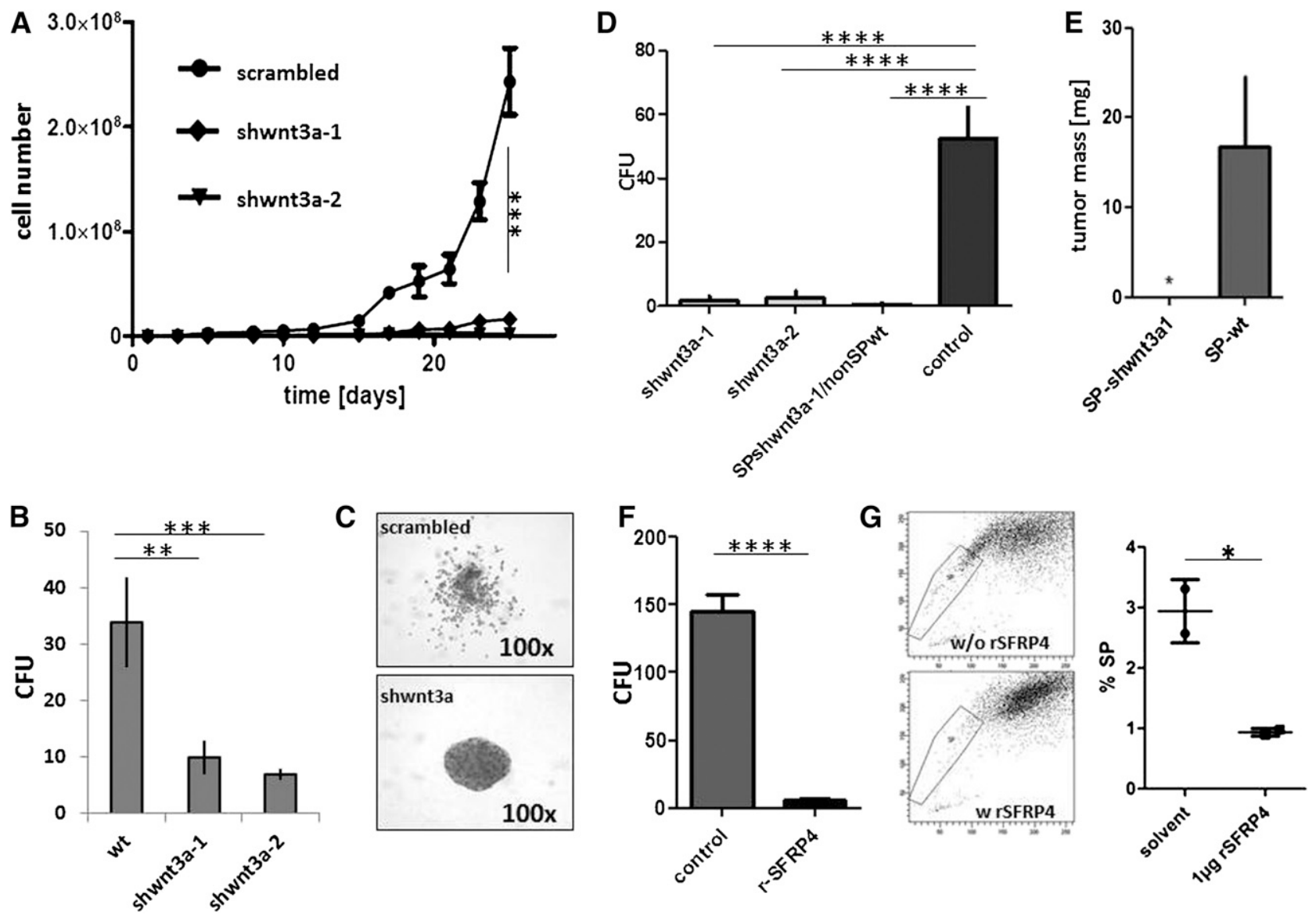

Figure 4. Reciprocal modulation of lymphoma growth by Wnt3a and SFRP4. (A-B) Lentiviral short hairpin (sh)-mediated silencing of Wnt3a decreased expansion capacity of OCI Ly3 lymphoma cells in suspension culture $(\mathrm{A})\left({ }^{* *} P<.001\right.$ after day 17,2 -way ANOVA) and reduced CFU after seeding $1 \times 10^{3}$ single cells in semisolid media (B) ${ }^{\star \star *} P<.001 ;{ }^{* \star \star} P<.0001$; 2-way ANOVA). (C) Outspread colony morphology of sh-scramble OCl-Ly3 controls, tight, vs condensed growth pattern of Wnt3a-silenced cells. (D) Reduced tumor formation from shWnt3a variants after implantation in ovo (2 left columns, ${ }^{* \star *} P<.0001$; 1 -way ANOVA). (E) Loss of tumorigenicity also after implantation of SP cells from the shWnt3a-1 variant cell line $\left(\mathrm{n}=3,{ }^{*} P=.048\right.$; paired Student $t$ test $)$ as well as from admixtures of SP cells of shWnt3a variant cells with wildtype non-SP cells (SP shWnt3a/nonSPwt: $4 \%$ SP cells, 96\% non-SP cells; D, third column; ${ }^{* \star \star \star} P<.0001 ; 1$-way ANOVA). (F-G) Recombinant SFRP4 reduced CFU activity from OCI Ly3 (F) $\left(2\right.$-sided Student $t$ test, $\left.{ }^{\star \star \star \star} P<.001\right)$ and reduced the proportion of SP cells in suspension culture $(\mathrm{G})(\mathrm{OCI}$ Ly3, 2-sided Student $t$ test, $P=.033)$.

found a significant stimulation of clonogenic growth of lymphoma SP and also, to some degree, non-SP cells (Figure 6A). Upon analyzing Wnt3a in this system, we found that Wnt3a was significantly enriched in the exosome fraction of the culture supernatant from OCI Ly 3 cells as well as from further lymphoma cell lines (Figure 6B). Exosomal Wnt3a migrated at a characteristic density of $1.16 \mathrm{~g} / \mathrm{mL}$ by sucrosegradient sedimentation (Figure 6C), and electron microscopy showed that Wnt3a was located on the outside of the limiting exosome membrane (Figure 6D). Functionally, purified lymphoma exosomes elicited Wnt activity in the TOP/FOP assay, and the activity was comparable to that of either ectopic Wnt3a expressed in the supernatant of L cells or that of purified recombinant Wnt3a (Figure 6E). Finally, we stained tumor tissue sections from 258 patients who were uniformly treated within the non-Hodgkin lymphoma B1 and B2 trials of the German High Grade Lymphoma Study Group $(\mathrm{DSHNHL})^{36,37}$ using an antibody against $\beta$-catenin. $\beta$-catenin localization was mostly confined to cytoplasm and exhibited an either a diffuse pattern or was found to be typically condensed at the perinuclear region (Figure 6F), which was similar to our findings in cell lines (supplemental Figure 7b). The proportion of $\beta$-catenin-positive tumor cells per sample varied significantly. In most cases, we detected $\beta$-catenin expression in $\sim 50 \%$ of the tumor cells (Figure 6G). With regard to the clinical outcome in this cohort of patients, whole $\beta$-catenin expression was not found to be associated with differences in overall survival or time to treatment failure after primary chemotherapy (supplemental Figure 10).

\section{Discussion}

The pivotal finding established in our study is the self-maintained intraclonal equilibrium of tumor cell populations. Components of this population equilibrium comprise not only classical motherdaughter relationships of SP and non-SP cells but also plasticity of cell types and a continuous exchange of Wnt signals between the DLBCL cells.

The concept of CSCs serves as an attractive model for explaining intratumoral heterogeneity, and tumor cells with SP phenotype appeared to follow this model. ${ }^{9,38}$ The findings of our study on aggressive lymphoma validated the characteristics of CSCs in SP cells at a single-cell level. However, in the exponentially growing tumor bulk, we observed not only expansion of SP cells but also a simultaneous direct conversion of non-SP cells back to the SP phenotype, thus contributing to tumor propagation. Congruently, similar transitions from CSCs to non-CSCs, and vice versa, have 
A

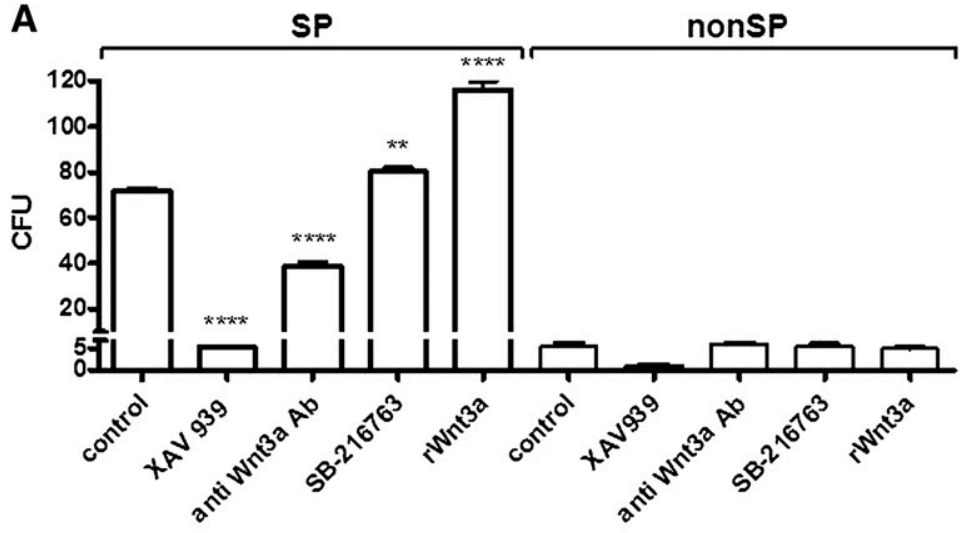

B

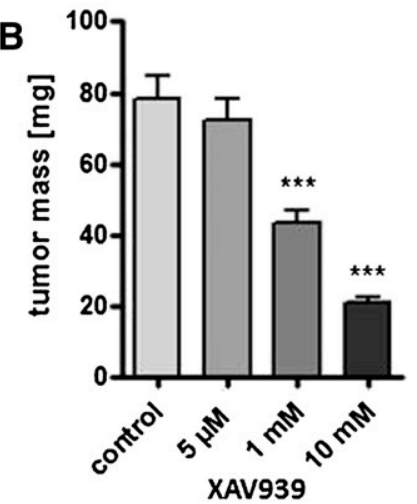

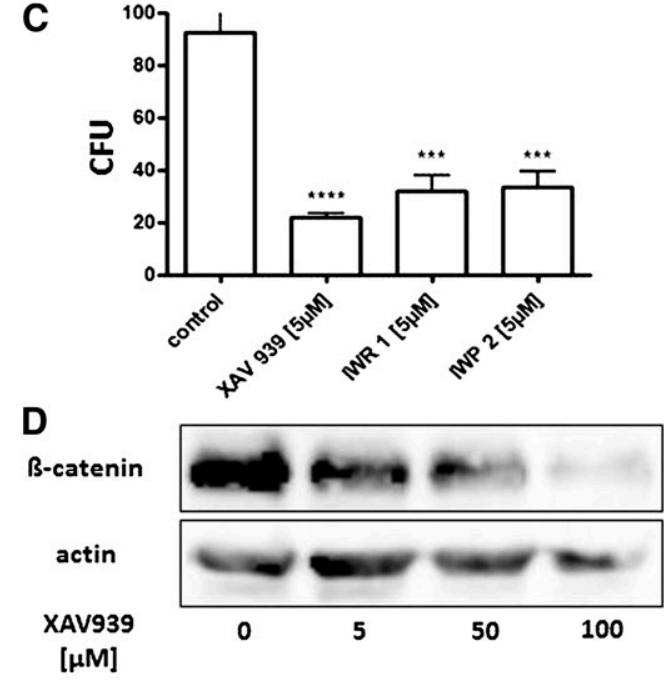

$\mathbf{E}$

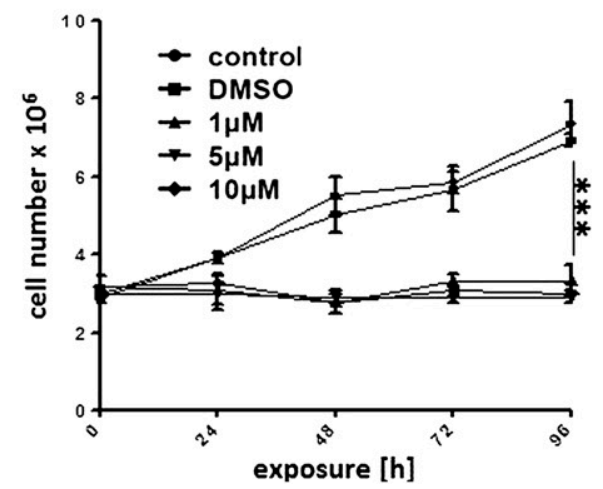

Figure 5. Pharmacologic inhibition of Wnt3a signaling impedes tumor growth of OCl-Ly3 lymphoma. (A) Exposure of $6 \times 10^{3}$ sorted OCI Ly3 SP- and $130 \times 10^{3}$ nonSP cells to different agents modulated the canonical wnt pathway, showing no effect on the rare colony formation from non-SP cells by any agent. Enhanced colony formation from SP cells was observed by recombinant Wnt3a (100 ng/mL) and the GSK-3- $\beta$ inhibitor SB-216763 (5 mM), in contrast to neutralizing antibody against Wnt3a $(20 \mu \mathrm{g} / \mathrm{mL})$ and tankyrase inhibitor XAV939 $(5 \mu \mathrm{M})$ suppressing colony formation from SP cells (1-way ANOVA, $\left.{ }^{* \star} P<.05,{ }^{* \star \star \star} P<0001\right)$. (B) When added to growing tumors in ovo, XAV939 reduced gross tumor growth (right; 1-way ANOVA, $P<.0001$ ), associated with an increasing loss of tumor cell infiltrates in the stroma of the implants (left; hematoxylin and eosin stain, original magnification $\times 100)$. (C) Equimolar concentrations of the wnt palmitoylation inhibitor IWP2 and the axin2 inhibitor IWR1 inhibited colony formation from $5 \times 10^{3}$ unsorted OCI Ly3 cells in semisolid media (1-way ANOVA, $\left.P<.0001\right)$. (D) Wnt inhibition by XAV939 reduced cellular $\beta$-catenin levels as detected by western blot. (E) XAV939 arrested cell proliferation compared with untreated or vehicle-treated controls (1-way ANOVA, $\left.{ }^{\star \star \star} P=.0041\right)$. DMSO, dimethylsulfoxide.

recently also been recognized in breast and bladder cancer cell line models. ${ }^{39,40}$ In our model, the SP and non-SP transitions were critically regulated by the tumor tissue context; ie, whether the cancer cells acted individually as a single cell or were functioning in close contact to neighboring cells. Importantly, the tissue context for either SP or non-SP cells was constituted not by exogenic stromal cells but by the respective population counterpart autonomous to the tumor clone. This self-organization of the tumor appears akin to the organizational pattern in bacteria termed "quorum sensing," where sensing bacterial density by metabolites or signal molecules regulates fate decisions of the whole population. ${ }^{41}$ In the present study, exosomal Wnt3a appeared to play the role of a quorumsensing factor, stimulating cell growth via locally increased concentrations, upon initiation of the clonal growth from individual colonies of SP cells expressing Wnt3a. Similarly, SFRP4 secreted from non-SP cells acted as a quorum-quenching factor, with a suppressive effect on lymphoma expansion.

Interestingly, when Wnt3a stimulation was blocked by genetic silencing or pharmacologic intervention, homeostasis was perturbed. Although inhibition of Wnt3a expression was compatible with immediate cell survival, the lymphoma cell cultures stagnated and eventually died. Some cells appeared to compensate for the loss of Wnt3a by generating very densely packed cell clones (Figure 4C), suggesting that direct cell contact or some factor in the tumor micromilieu might have partially compensated the loss of Wnt3a. Overall, SP cells displayed a dual function in the propagation of lymphoma bulk. Besides generating progeny, the SP cells provided Wnt3a to surrounding non-SP cells. Therefore, our study confirmed that tumor population expansion represents not a stochastic growth from independent cells but rather a deterministic process, where fate decisions depend on the cellular neighborhood. ${ }^{42}$

Activation of Wnt-pathway components has previously been described in several other malignancies, including colorectal cancer, where APC mutants cause a strong activation of the canonical Wnt pathway. ${ }^{13}$ In contrast to the cases of APC-mutated colorectal cancer, the effects between lymphoma subpopulations appear more subtle, as evidenced by the strong nuclear expression of $\beta$-catenin in colorectal cancer when compared with the predominant cytoplasmic and perinuclear $\beta$-catenin staining in the lymphomas, similar to the situation in multiple myeloma. ${ }^{43}$ Similar to us, Ge et al recently detected $\beta$-catenin expression in most DLBCL samples and described a correlation of increased nuclear $\beta$-catenin levels with 


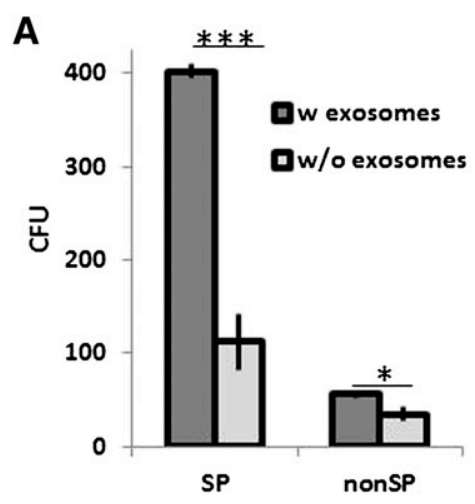

B

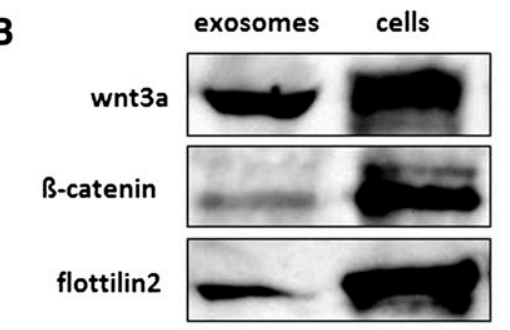

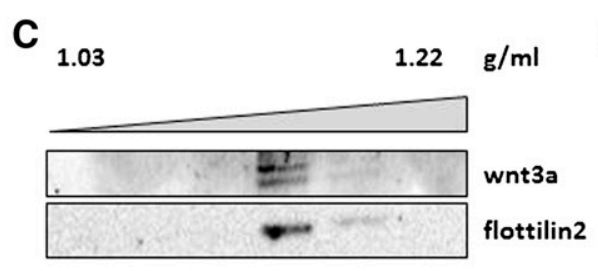

E

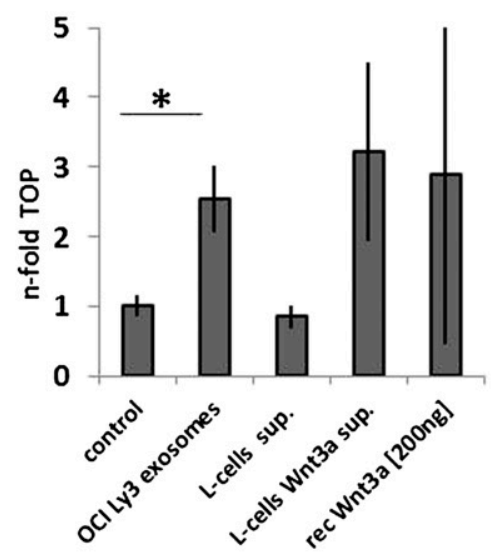

$\mathbf{F}$

\section{G}

D
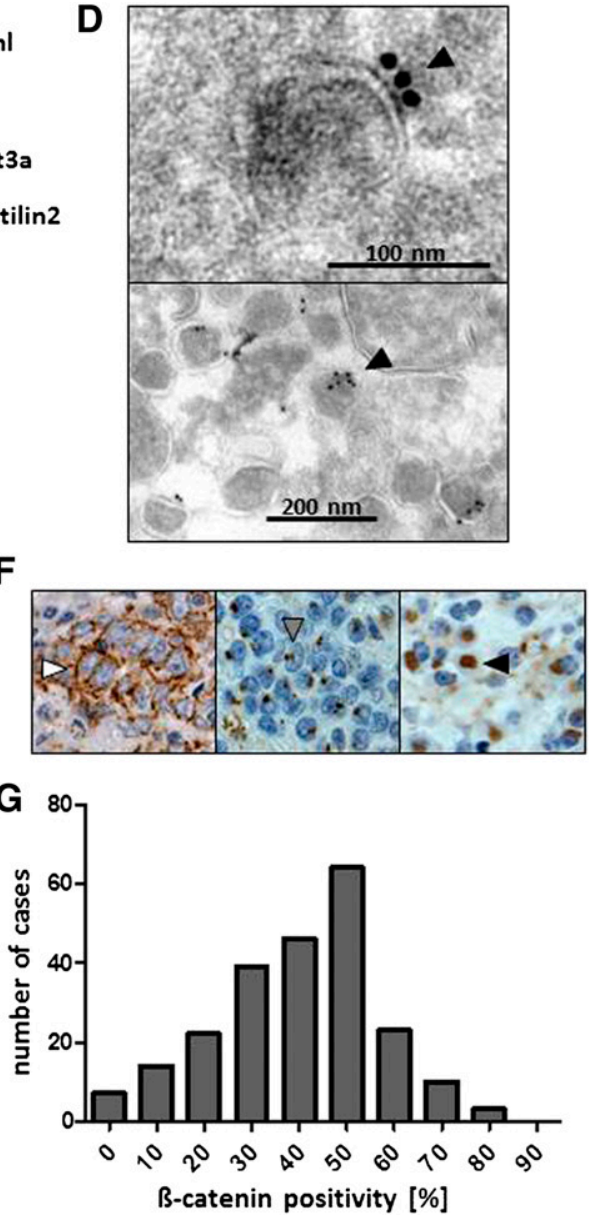

Figure 6. Exosome-mediated Wnt3a signal transmission and $\boldsymbol{\beta}$-catenin in clinical samples of DLBCL. (A) Enhanced CFU activity of OCI Ly3 SP cells by addition of autologous exosomes $(2 \mathrm{mg} / \mathrm{mL})$ in semisolid media $\left(\mathrm{n}=3,{ }^{\star \star \star} P<.0001 ;{ }^{\star} P=.046 ; 2\right.$-sided Student $t$ test). (B) Enrichment of Wnt3a in the exosome fraction of lymphoma cell line supernatants (OCl Ly3, upper panel; further cell lines, lower panel; flottilin2 as an exosome marker). (C) Sedimentation of Wnt3a at a density of $1.16 \mathrm{~g} / \mathrm{mL}$ together with flottillin2 in a sucrose gradient of OCl Ly3 supernatant. (D) Electron microscopy with immunogold staining showing Wnt3a at the surface of the exosomes (filled arrowhead, upper panel), with heterogeneous amounts between exosomes (lower panel). (E) Increased TOPflash (TOP) reporter activity by exosomes from OCI Ly3 lymphoma cells (2.3-fold, ${ }^{*} P=.048,2$-sided $t$ test), comparable to supernatant of $\mathrm{L}$ cells with ectopic Wnt3a expression or recombinant Wnt3a (representative example of 3 independent experiments). (F) Detection of $\beta$-catenin in aggressive lymphoma samples with cytoplasmic staining (right panel, empty arrowhead), perinuclear staining in most cases (middle panel, gray arrowhead), and rarely nuclear staining (right panel, black arrowhead; original magnification $\times 400$; compare with supplemental Figure 10). (G) In the samples of the prospective DSHNHL B1/B2 protocols $(n=258)$, the proportions of positive cells per sample showed a unimodal distribution of $\beta$-catenin expression levels (compare with supplemental Figure 10B).

clinically advanced-stage disease. ${ }^{44}$ In our cohort of patients treated in the prerituximab era, we did not detect an association between the amount of $\beta$-catenin-positive cells per sample and patient prognosis (supplemental Figure 10). Measuring whole $\beta$-catenin integrates both $\beta$-catenin activation and $\beta$-catenin decay, thus providing evidence for involvement of canonical Wnt signaling only. ${ }^{13}$ For an estimate of an association between prognosis and $\beta$-catenin activation levels, studies of nonphosphorylated $\beta$-catenin or canonical wnt targets may be warranted.

In the present study, Wnt $3 a$ and SFRP4 expression in DLBCL SP and non-SP cells was regulated by an epigenetic mechanism, wherein a differential cytosine guanine dinucleotide island methylation of the respective promoters was noted. Previous studies on acute leukemia demonstrated the promoter methylation patterns of the Wnt pathway genes in the whole tumor sample, emphasizing on the differences between individual tumors. ${ }^{17,18}$ Our finding of intratumoral heterogeneity in lymphomas dictates that even epigenetic changes of Wnt pathway components in quantitatively minor subpopulations such as SP cells might be relevant once they become critical for the tumor infrastructure.
Upon analysis of the extracellular route of lymphoma-derived Wnt3a, we discovered enrichment in the exosome fraction of the cell supernatant. Exosomes or exosome-like vesicles have already been described to transfer Wnt proteins, in particular in niches requiring signal transmission across short distance, such as the neuromuscular synapse or as so-called argosomes at the basolateral imaginal disc epithelium of Drosophila. ${ }^{21,23}$ The communication between neighboring cells in the tumor micromilieu may represent a similar situation, in which exosomes facilitate exchange of autocrine or paracrine Wnt proteins. Although our results clearly document Wnt3a synthesis and secretion from lymphoma cells, the precise mechanism directing Wnt3a to the limiting exosome membrane is not yet known. In Drosophila, recent studies unraveled that several key proteins involved in MVB formation as well as R-SNARE Ykt6 were required for proper exosomal Wnt signal transmission. ${ }^{25,45}$ Similar mechanisms may also pertain to hematopoietic malignancies such as leukemias and lymphomas, for which we recently found the $\mathrm{ABC}$ transporter $\mathrm{A} 3$ to participate in a common apparatus of MVB formation and exosome secretion. ${ }^{29,46}$ 
How do our findings integrate in current concepts of lymphoma pathobiology, in particular DLBCL? At first sight, the CSC concept might not appear intuitive for malignant entities derived from mature, postgerminal B cells such as DLBCL. Indeed, lymphoma SP and non-SP cells expressed mature B-cell markers such as CD19 at equal levels and did not differ in the expression levels of HSC markers. Therefore, we view the genes associated with the functional state of SP, including increased Wnt $3 a$, as a resurgence of an oncofetal program on the background of mature B cells. In recent years, gene expression profiling has provided significant knowledge about the heterogeneity of DLBCL, in particular the distinction of ABC type vs the GCB type and primary mediastinal B-cell lymphoma, or the characterization of molecular Burkitt lymphoma. ${ }^{47-49}$ Within the panel of cell lines and the cases of primary aggressive lymphoma samples in this study, we have not noted a segregation of SP formation or differential Wnt-pathway member expression associated with ABCvs GCB-type assignment. Thus, we propose that the formation of SP and non-SP cells and, in particular, their mutual functional interaction through Wnt signaling are a common feature of lymphoma infrastructure, imposed on DLBCL of different subtypes. In a therapeutic perspective, this biological feature may allow the identification of targets for intervention, such as pharmacologic silencing of Wnt3a expression, application of Wnt antagonists, or interference with downstream mediators of the wnt pathways by molecules such as tankyrase inhibitors.

Taken together, the results indicate that DLBCL possess a selforganized infrastructure comprising SP and non-SP cells, where conversion of cell states is modulated by exosome-mediated Wnt signaling.

\section{Acknowledgments}

The authors thank Jörg Wilting for support with the CAM assays and Perri Hartenstein as well as Editage services for linguistic editing.

This work was supported by the Deutsche Forschungsgemeinschaft (DFG Wu 310/3-1) (G.G.W.) and by the University Medicine Goettingen (Jacob-Henle program grant) (T.A.) (Forschungsförderung) (R.K.).

\section{Authorship}

Contribution: M.D. and R.K. designed and performed research, analyzed data, and contributed to the writing of the paper; T.A., N.D., B.C, A.C., D.W., M.L., A.G., C.K., S.B., T.H., V.V., and W.K. performed research; L.O. analyzed data; M.Z. contributed clinical data sets; L.T. contributed support and detailed discussions; and G.G.W. designed research, analyzed data, and wrote the paper. Flow cytometry and fluorescence-activated cell sorter and cell sorting were performed in the Flow Cytometry Core Facility at the University of Goettingen.

Conflict-of-interest disclosure: The authors declare no competing financial interests.

Correspondence: Gerald Wulf, Department of Hematology and Oncology, Georg-August-University Goettingen, RobertKoch-Str.40, 37075 Goettingen, Germany; e-mail: gwulf@med. uni-goettingen.de.

\section{References}

1. Marusyk A, Polyak K. Tumor heterogeneity: causes and consequences. Biochim Biophys Acta. 2010;1805(1):105-117.

2. Brock $A$, Chang $H$, Huang $S$. Non-genetic heterogeneity - a mutation-independent driving force for the somatic evolution of tumours. Nat Rev Genet. 2009;10(5):336-342.

3. Bruce WR, Van Der Gaag H. A quantitative assay for the number of murine lymphoma cells capable of proliferation in vivo. Nature. 1963;199:79-80.

4. Ogawa M, Fried J, Sakai Y, Strife A, Clarkson BD. Studies of cellular proliferation in human leukemia. VI. The proliferative activity, generation time, and emergence time of neutrophilic granulocytes in chronic granulocytic leukemia. Cancer. 1970;25(5):1031-1049.

5. Bonnet D, Dick JE. Human acute myeloid leukemia is organized as a hierarchy that originates from a primitive hematopoietic cell. Nat Med. 1997;3(7):730-737.

6. Al-Haij M, Wicha MS, Benito-Hernandez A, Morrison SJ, Clarke MF. Prospective identification of tumorigenic breast cancer cells. Proc Natl Acad Sci U S A. 2003;100(7):3983-3988.

7. Goodell MA, Brose K, Paradis G, Conner AS, Mulligan RC. Isolation and functional properties of murine hematopoietic stem cells that are replicating in vivo. J Exp Med. 1996;183(4): 1797-1806.

8. Wulf GG, Wang RY, Kuehnle I, et al. A leukemic stem cell with intrinsic drug efflux capacity in acute myeloid leukemia. Blood. 2001;98(4):1166-1173.

9. Hirschmann-Jax C, Foster AE, Wulf GG, et al. A distinct "side population" of cells with high drug efflux capacity in human tumor cells. Proc Natl Acad Sci U S A. 2004;101(39):14228-14233.
10. Moserle L, Ghisi M, Amadori A, Indraccolo S. Side population and cancer stem cells: therapeutic implications. Cancer Lett. 2010;288(1):1-9.

11. Lee MR, Ju HJ, Kim BS, Ko YH, Kim WS, Kim SJ. Isolation of side population cells in B-cell nonHodgkin's lymphomas. Acta Haematol. 2013; 129(1):10-17.

12. Siegfried E, Perrimon N. Drosophila wingless: a paradigm for the function and mechanism of Wnt signaling. Bioessays. 1994;16(6):395-404

13. Willert K, Nusse R. Beta-catenin: a key mediator of Wnt signaling. Curr Opin Genet Dev. 1998;8(1) 95-102.

14. Morin PJ, Sparks AB, Korinek V, et al. Activation of beta-catenin-Tcf signaling in colon cancer by mutations in beta-catenin or APC. Science. 1997; 275(5307):1787-1790.

15. Rubinfeld B, Albert I, Porfiri E, Fiol C, Munemitsu S Polakis P. Binding of GSK3beta to the APC-betacatenin complex and regulation of complex assembly. Science. 1996;272(5264):1023-1026.

16. Van Den Berg DJ, Sharma AK, Bruno E, Hoffman $R$. Role of members of the Wnt gene family in human hematopoiesis. Blood. 1998;92(9): 3189-3202.

17. Reya $T$, Duncan AW, Ailles $L$, et al. A role for $W n t$ signalling in self-renewal of haematopoietic stem cells. Nature. 2003;423(6938):409-414.

18. Valencia A, Román-Gómez J, Cervera J, et al. Wnt signaling pathway is epigenetically regulated by methylation of Wnt antagonists in acute myeloid leukemia. Leukemia. 2009;23(9): 1658-1666.

19. Willert K, Brown JD, Danenberg E, et al. Wnt proteins are lipid-modified and can act as stem cell growth factors. Nature. 2003;423(6938): 448-452.
20. Verras M, Papandreou I, Lim AL, Denko NC Tumor hypoxia blocks Wnt processing and secretion through the induction of endoplasmic reticulum stress. Mol Cell Biol. 2008;28(23): 7212-7224.

21. Greco V, Hannus M, Eaton S. Argosomes: a potential vehicle for the spread of morphogens through epithelia. Cell. 2001;106(5):633-645.

22. Panáková D, Sprong H, Marois E, Thiele C, Eaton S. Lipoprotein particles are required for Hedgehog and Wingless signalling. Nature. 2005;435(7038): 58-65.

23. Korkut C, Ataman B, Ramachandran P, et al. Trans-synaptic transmission of vesicular Wnt signals through Evi/Wntless. Cell. 2009;139(2): 393-404.

24. Mulligan KA, Fuerer $C$, Ching W, Fish M, Willert $K$, Nusse R. Secreted Wingless-interacting molecule (Swim) promotes long-range signaling by maintaining Wingless solubility. Proc Natl Acad Sci U S A. 2012;109(2):370-377.

25. Gross JC, Chaudhary V, Bartscherer K, Boutros M. Active Wnt proteins are secreted on exosomes. Nat Cell Biol. 2012;14(10):1036-1045

26. Février B, Raposo G. Exosomes: endosomalderived vesicles shipping extracellular messages. Curr Opin Cell Biol. 2004;16(4):415-421.

27. Pan BT, Teng K, Wu C, Adam M, Johnstone RM. Electron microscopic evidence for externalization of the transferrin receptor in vesicular form in sheep reticulocytes. J Cell Biol. 1985;101(3): 942-948.

28. Raposo G, Nijman HW, Stoorvogel W, et al. $B$ lymphocytes secrete antigen-presenting vesicles. J Exp Med. 1996;183(3):1161-1172.

29. Aung T, Chapuy B, Vogel D, et al. Exosomal evasion of humoral immunotherapy in aggressive B-cell lymphoma modulated by ATP-binding 
cassette transporter A3. Proc Natl Acad Sci U S A. 2011;108(37):15336-15341.

30. Lok MS, Koshiba H, Han T, Abe S, Minowada J, Sandberg AA. Establishment and characterization of human B-lymphocytic lymphoma cell lines (BALM-3, -4 and -5); intraclonal variation in the B-cell differentiation stage. Int J Cancer. 1979; 24(5):572-578.

31. Tweeddale $M$, Jamal N, Nguyen A, Wang $X H$ Minden MD, Messner HA. Production of growth factors by malignant lymphoma cell lines. Blood. 1989;74(2):572-578.

32. Yee $\mathrm{C}$, Biondi $\mathrm{A}$, Wang $\mathrm{XH}$, et al. A possible autocrine role for interleukin- 6 in two lymphoma cell lines. Blood. 1989;74(2):798-804

33. Steemers FJ, Chang W, Lee G, Barker DL, Shen $\mathrm{R}$, Gunderson KL. Whole-genome genotyping with the single-base extension assay. Nat Methods. 2006;3(1):31-33.

34. Diering N, Aung T, Chapuy B, et al. Putative tumor stem cells of SP phenotype in aggressive B-cell lymphoma. Onkologie. 2010;33(suppl 6):188.

35. Chambers SM, Boles NC, Lin KY, et al. Hematopoietic fingerprints: an expression database of stem cells and their progeny. Cell Stem Cell. 2007;1(5):578-591.

36. Pfreundschuh $M$, Trümper L, Kloess $M$, et al; German High-Grade Non-Hodgkin's Lymphoma Study Group. Two-weekly or 3-weekly CHOP chemotherapy with or without etoposide for the treatment of elderly patients with aggressive lymphomas: results of the NHL-B2 trial of the DSHNHL. Blood. 2004;104(3):634-641.

37. Pfreundschuh M, Schubert J, Ziepert M, et al. Two-weekly or 3-weekly CHOP chemotherapy with or without etoposide for the treatment of young patients with good-prognosis (normal LDH) aggressive lymphomas: results of the NHL-B1 trial of the DSHNHL. Lancet Oncol. 2008;9(2): 105-116.

38. Magee JA, Piskounova E, Morrison SJ. Cance stem cells: impact, heterogeneity, and uncertainty. Cancer Cell. 2012;21(3):283-296.

39. Chaffer CL, Brueckmann I, Scheel C, et al. Normal and neoplastic nonstem cells can spontaneously convert to a stem-like state. Proc Natl Acad Sci U S A. 2011;108(19):7950-7955.

40. He K, Xu T, Goldkorn A. Cancer cells cyclically lose and regain drug-resistant highly tumorigenic features characteristic of a cancer stem-like phenotype. Mol Cancer Ther. 2011;10(6): 938-948.

41. Li Z, Nair SK. Quorum sensing: how bacteria can coordinate activity and synchronize their response to external signals? Protein Sci. 2012;21(10): 1403-1417

42. Snijder B, Pelkmans L. Origins of regulated cellto-cell variability. Nat Rev Mol Cell Biol. 2011; 12(2):119-125.

43. Sukhdeo K, Mani M, Hideshima T, et al. $\beta$-catenin is dynamically stored and cleared in multiple myeloma by the proteasome-aggresome- autophagosome-lysosome pathway. Leukemia. 2012;26(5):1116-1119.

44. Ge X, Lv X, Feng L, Liu X, Wang X. High expression and nuclear localization of $\beta$-catenin in diffuse large B-cell lymphoma. Mol Med Rep. 2012;5(6):1433-1437.

45. Koles K, Nunnari J, Korkut C, et al. Mechanism of evenness interrupted (Evi)-exosome release at synaptic boutons. J Biol Chem. 2012;287(20): 16820-16834.

46. Chapuy B, Koch R, Radunski U, et al. Intracellular $A B C$ transporter $A 3$ confers multidrug resistance in leukemia cells by lysosomal drug sequestration. Leukemia. 2008;22(8):1576-1586.

47. Alizadeh AA, Eisen MB, Davis RE, et al. Distinct types of diffuse large B-cell lymphoma identified by gene expression profiling. Nature. 2000; 403(6769):503-511.

48. Rosenwald A, Wright G, Leroy K, et al. Molecular diagnosis of primary mediastinal $B$ cell lymphoma identifies a clinically favorable subgroup of diffuse large $B$ cell lymphoma related to Hodgkin lymphoma. J Exp Med. 2003;198(6):851-862.

49. Hummel M, Bentink S, Berger $\mathrm{H}$, et al; Molecular Mechanisms in Malignant Lymphomas Network Project of the Deutsche Krebshilfe. A biologic definition of Burkitt's lymphoma from transcriptional and genomic profiling. N Engl J Med. 2006;354(23):2419-2430. 